\title{
A new heterochromatin banding pattern revealed by modified HKG banding technique in maize chromosomes
}

\author{
CARLOS ROBERTO DE CARVALHO* \& LUIZ SÉRGIO SARAIVA \\ Departamento de Biologia Geral, Universidade Federal de Viçosa, 36570-000, Viçosa-MG, Brazil.
}

\begin{abstract}
A new heterochromatin banding pattern for maize root tip chromosomes was revealed by a modified HKG- ( $\mathrm{HCl}-\mathrm{KOH}-$ Giemsa) banding technique. Comparison of this pattern with that obtained by the $\mathrm{C}$-banding technique showed that the two techniques stain different types of heterochromatin. The HKG-banding technique stains both centromeric and intercalary heterochromatin, whereas the C-banding technique reveals only knob heterochromatin. The presence of an HKG intercalary band adjacent to the knob suggests a more complex constitution for the heterochromatic block of the knob.
\end{abstract}

Keywords: C-banding, centromeric heterochromatin, HKG-banding, knob heterochromatin, maize chromosomes, maize cytogenetics.

\section{Introduction}

The C-banding technique has been used extensively to reveal constitutive heterochromatin in maize root tip chromosomes, and the results show that different classes of maize heterochromatin can be differentiated through this procedure (Aguiar-Perecin, 1985).

Cytologically, maize heterochromatin was classified into five types: (1) centromeric heterochromatin; (2) NOR heterochromatin; (3) knob heterochromatin, present in the standard (A) chromosomes; (4) centromeric knob-like heterochromatin; and (5) distal heterochromatin, present in the $\mathrm{B}$ chromosomes (for review see Carlson, 1988). However, inconsistency of stainability in obtaining $\mathrm{C}$-band involving centromeric A heterochromatin has been reported (Hadlaczky \& Kalman, 1975; Ward, 1980; Chow \& Larter, 1981; Mastenbroek \& De Wet, 1983; Aguiar-Perecin, 1985). The difficulty in obtaining clear and defined centromeric bands in comparison to the knob dark bands has been explained by differential responses to different $\mathrm{C}$-banding procedures. It has also been suggested that centromeric $\mathrm{A}$ and knob heterochromatin are different types of heterochromatin (Ward, 1980; Chow \& Larter, 1981; Aguiar-Perecin, 1985). This difference was demonstrated at the molecular level by cloning and in-situ hybridization techniques. The 185-bp highly repetitive DNA sequence $\left(\mathrm{H}_{2}\right.$ sequence $)$ is a major

${ }^{*}$ Correspondence. component of knob heterochromatin of A chromosomes and centromeric heterochromatin of the B chromosome. This sequence is not present in other heterochromatic segments of maize chromosomes and probably other highly repetitive DNA sequences are present in centromeric heterochromatin (Peacock et al., 1981; Viotti et al., 1985).

Peacock et al. (1981) proposed that the neocentric response, observed during meiosis in the presence of abnormal chromosome 10 , is a consequence of the DNA of knob heterochromatin. In addition, Viotti et $a l$. (1985) suggested that the DNA of knob heterochromatin must contain one or more centromeric sequences which may be interspersed with the highly repeated $\mathrm{H}_{2}$ sequence, indicating a possible functional role in the neocentric activity.

The C-banding technique for maize somatic chromosomes has also revealed, although in low frequency, the presence of additional interstitial or intercalary bands (Chow \& Larter, 1981; Khoung \& Schubert, 1986). However, these bands have not been demonstrated by other authors working with maize. These intercalary bands have not been used for the maize heterochromatin classification suggested by Carlson (1988).

In this paper, the HKG (HCl-KOH-Giemsa)banding technique developed for wheat chromosomes (Shang et al., 1988) was modified to obtain welldefined centromeric and intercalary bands for mitotic maize chromosomes. A short $\mathrm{C}$-banding technique was 
also developed to show the regions corresponding to the knobs, and the banding patterns from both techniques were compared.

To produce chromosome slides suitable for clear HKG and C-banding patterns, a series of preliminary technical procedures were performed.

\section{Materials and methods}

\section{Plant material}

Inbred stocks of maize (Zea mays L.) derived from three cycles of selfing Dent and Flint composites were used in the present investigation. Seeds of inbreds L-SRR-D-86-19 and L-SRR-F-84-5 were obtained from Federal University of Viçosa (Brazil).

\section{Pre-treatment and fixation}

Seeds were germinated in agar plates with a film of distilled water in the dark at $32^{\circ} \mathrm{C}$ for $26-28 \mathrm{~h}$, and then treated with a 0.05 per cent 8 -hydroxyquinoline solution (Merck) for $2 \mathrm{~h} 30 \mathrm{~min}$ at $32^{\circ} \mathrm{C}$. The embryos were removed from the seeds, washed with distilled water and fixed in fresh cold methanol-acetic acid (3:1). The fixative was changed three times at $15 \mathrm{~min}$ intervals and then stored in the freezer $\left(-20^{\circ} \mathrm{C}\right)$ for one to several days.

\section{Enzymatic maceration}

Approximately 20 fixed embryos were washed in tap water for $5 \mathrm{~min}$ and then in distilled water. Meristemcontaining primary root (radicle) tips and adventitious seminal root regions were removed from the embryos and transferred to $1 \mathrm{ml}$ of distilled water in a depression well (e.a. $25 \mathrm{~mm}$ diameter) of an agglutination slide. By using micro-ophthalmological scissors, the root-tips were minced in several tiny fragments and then transferred with a Pasteur pipette to a $15 \mathrm{ml}$ centrifuge tube containing $3 \mathrm{ml}$ of distilled water. After centrifugation $(150 \mathrm{~g}$ for $2 \mathrm{~min}$ ), the pellet was resuspended in $2 \mathrm{ml}$ of freshly prepared enzyme solution, consisting of $0.2 \mathrm{ml}$ flaxzyme (NOVO) plus $1.6 \mathrm{ml}$ distilled water, and incubated at $35^{\circ} \mathrm{C}$ for $2 \mathrm{~h} 30 \mathrm{~min}$. The supernatant was then removed by centrifugation $(150 \mathrm{~g}$ for $1 \mathrm{~min})$, the pellet was gently resuspended in $2 \mathrm{ml}$ distilled water and filtered through a $60 \mu \mathrm{m}$ nylon mesh into another centrifuge tube. The volume was completed to $5 \mathrm{ml}$ with distilled water.

\section{Slide preparations}

After centrifugation ( $150 \mathrm{~g}$ for $1 \mathrm{~min}$ ), the pellet was washed once with fresh cold methanol-acetic acid
(3:1), and then resuspended in $0.5 \mathrm{ml}$ of the same fixative. Four to five drops of cell suspension were dropped on to a slide covered with a film of cold 45 per cent acetic acid. The drops were delivered from a Pasteur pipette $30-40 \mathrm{~cm}$ above the slide. The slide was then air-dried by fast wave movements and placed on to a hot-plate (surface temperature $50^{\circ} \mathrm{C}$ ) for 10 min, and aged in an incubator at $35^{\circ} \mathrm{C}$ for one to several days.

\section{Modified C-banding procedure}

Two-week-old slides were treated with freshly prepared 5 per cent $\mathrm{Ba}(\mathrm{OH})_{2} .8 \mathrm{H}_{2} \mathrm{O}$ solution at $56^{\circ} \mathrm{C}$, with continuous agitation for $10-15 \mathrm{~s}$, washed in 70 per cent ethanol (two changes), and then in absolute ethanol, and transferred to methanol-acetic acid $(8: 1)$. The slides were dried on a hot-plate for a few minutes and stained with 3 per cent Giemsa (MERCK) in phos-

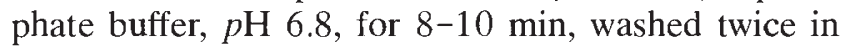
distilled water and air dried.

\section{HKG-banding}

The procedure of Shang et al. (1988) was modified to produce $\mathrm{HKG}$-bands for maize chromosomes. One- to five-day-old slides were hydrolysed in $1 \mathrm{~N} \mathrm{HCl}$ at $60^{\circ} \mathrm{C}$ for 4-6 min and washed four times in distilled water for a total of $10 \mathrm{~min}$. The slides were briefly immersed in 0.9 per cent $\mathrm{NaCl}$, plunged 10 times in 70 per cent ethanol, dried on a hot-plate (surface temperature $50^{\circ} \mathrm{C}$ ) for a few seconds and immersed immediately in a fresh $0.06 \mathrm{~N} \mathrm{KOH}$ solution for 8-12 s with continuous agitation, at room temperature. The slides were washed in 70 per cent ethanol (two changes), absolute ethanol and transferred to methanol-acetic acid $(8: 1)$. They were then dried on a hot-plate for a few minutes and stained in the same way as described for the modified C-banding procedure.

\section{Photography}

Mitotic figures from HKG- and C-banding slides were photographed with a $\mathrm{BH}-2$ Olympus photomicroscope equipped with a $100 \times$ NSC plan Apo oil objective and a $5 \times$ L NFK eyepiece. The film (Agfa Copex Pan-Tri13) was developed in D-76 (KODAK) for $14 \mathrm{~min}$, at $20^{\circ} \mathrm{C}$. Prints were made on Kodacrome print-RC F- 3 in 1:3 DEKTOL (KODAK).

\section{Results and discussion}

By using the methodology described in this paper, it was possible to obtain good chromosome preparations for subsequent banding treatments. Chromosomes 
were well spread in the same plane of focus, showing neither cytoplasmic background nor residue, with the morphology well preserved in different mitotic phases (Fig. 1).

The modified HKG-banding (Fig. 1a,b,c) and the short C-banding procedures (Fig. 1d) revealed two distinct banding patterns, both with well-resolved dark bands.

The HKG-banding patterns revealed two basic band types: the centromeric with dark bands strongly stained on one or both sides of the primary constriction, and the intercalary or interstitial type located between centromeric and terminal positions. Most of these intercalary bands were located adjacent to knob posi- tions. This was revealed by the differential staining of the dark HKG intercalary bands and the knob staining vestige present in some HKG-banding preparations (Fig. 1a,b).

The HKG-banding patterns were consistent not only at metaphase (Fig. 1b) but also during other phases of mitosis such as prophase (Fig. 1a) and anaphase (Fig. 1c). By using this HKG-banding technique it was possible to construct high-resolution caryograms revealing homomorphic and heteromorphic HKGbands in metaphase chromosomes (Fig. 2).

Our short C-banding technique revealed heavily stained distal C-bands at the well-known knob heterochromatin positions as described in previous reports.
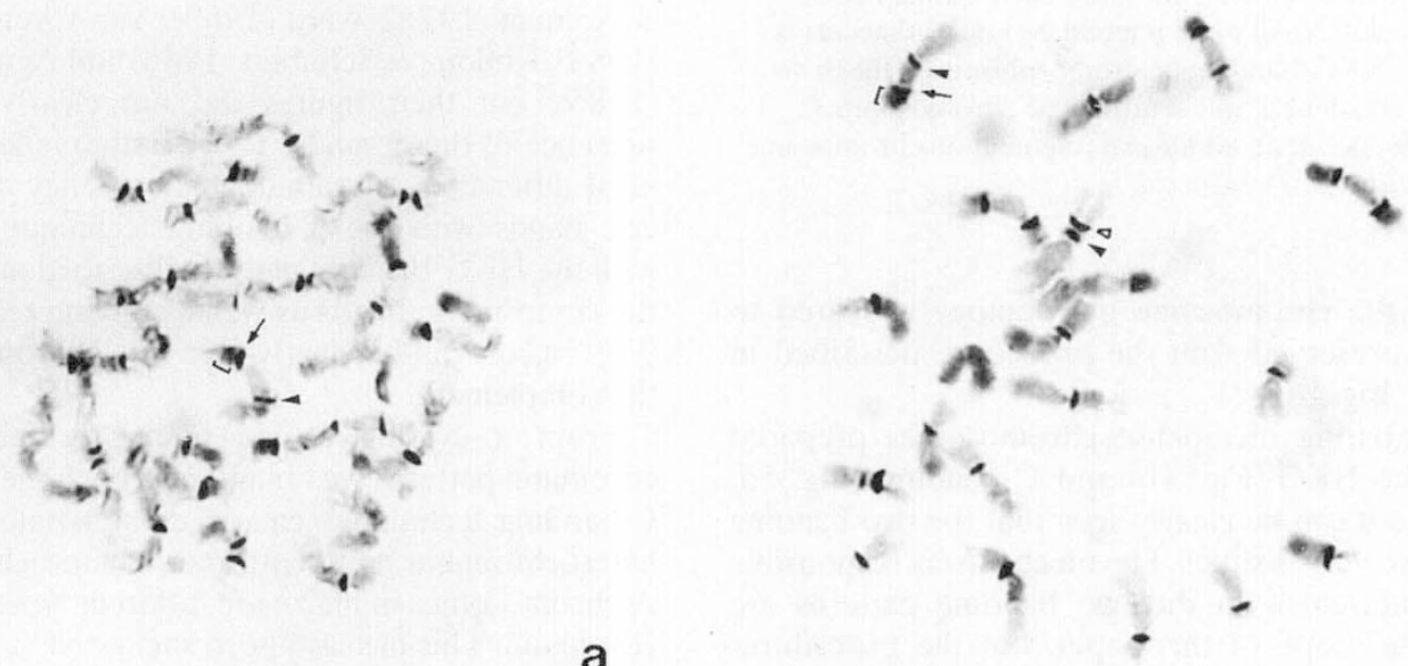

a
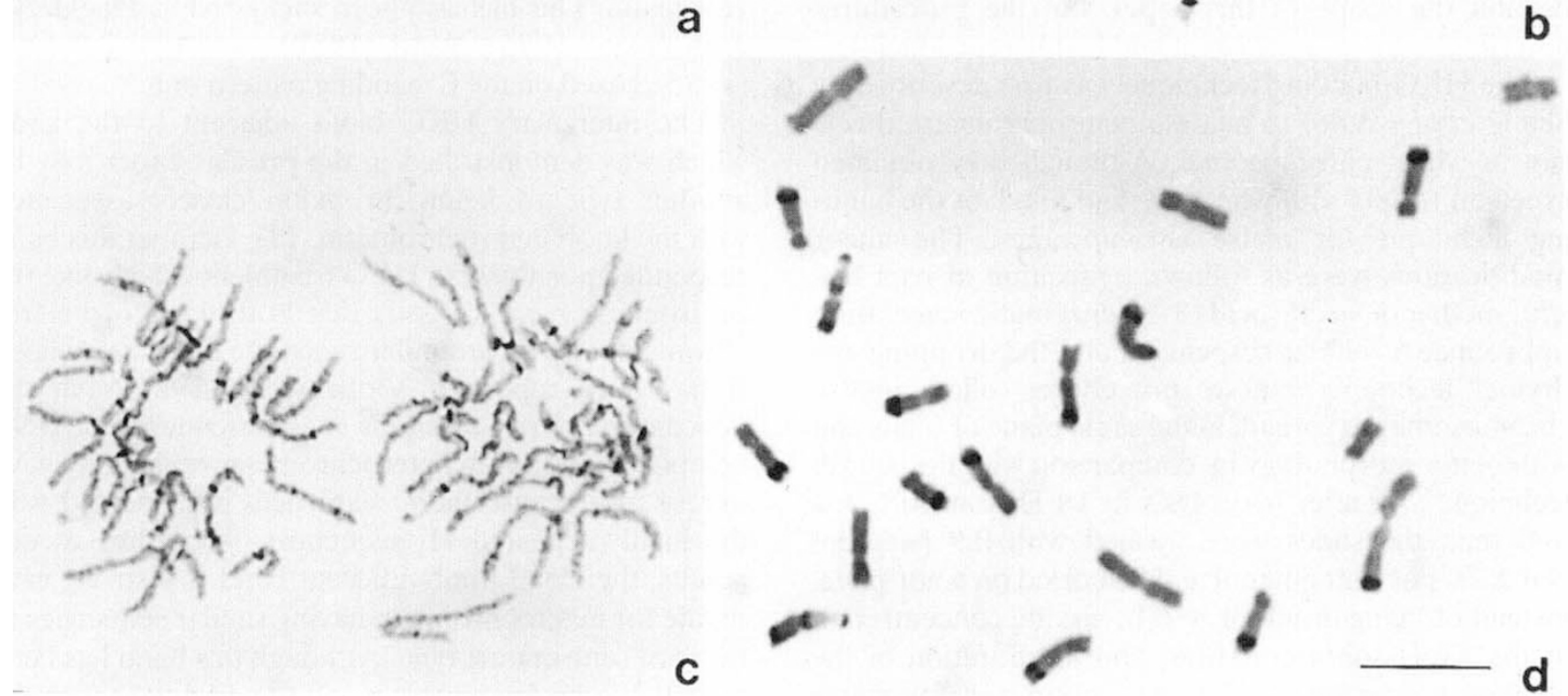

Fig. 1 The HKG-banding (a,b,c) and C-banding (d) patterns of root tip maize chromosomes $(2 n=20)$. Bar $=10 \mu \mathrm{m}$. (a) Prophase and (b) metaphase showing centromeric (filled arrowheads), intercalary (open arrowheads) and intercalary knob-adjacent bands (arrows). The brackets indicate a knob vestige. (c) Anaphasic chromosomes with one to five HKG-bands. (d) C-banding metaphasic chromosomes with bands stained only at the knob regions. 


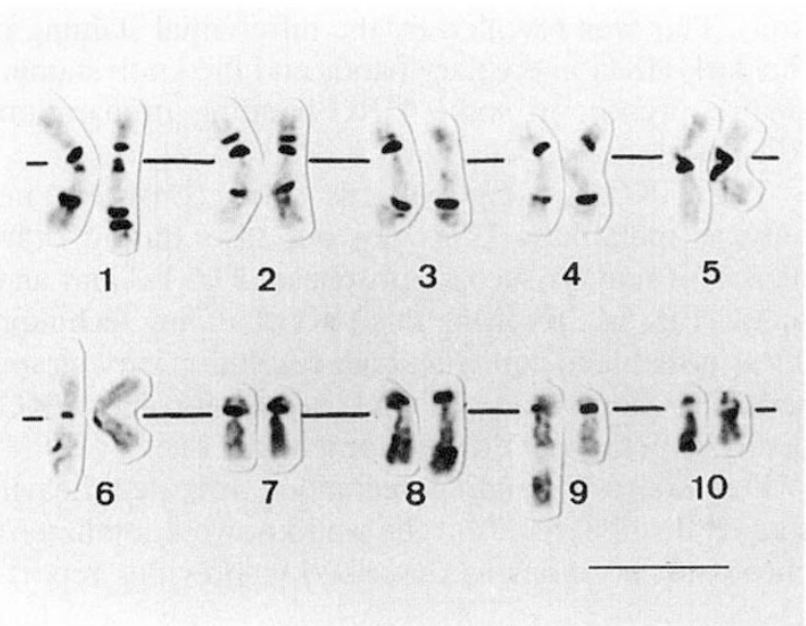

Fig. 2 HKG-banded maize karyotype $(2 n=20)$. Homologous chromosome showing the close correspondence of centromeric, intercalary and intercalary knob-adjacent bands. Note HKG-banding heteromorphisms on the distal part of chromosome 1 and centromeric chromosome 3 regions. Presence of an additional fragment on chromosome 9. $\mathrm{Bar}=10 \mu \mathrm{m}$.

However, the chromosome morphology appeared to be better preserved with the procedure described in this paper (Fig. 1d).

By comparing metaphase chromosomes prepared through the HKG-(Fig. 1b) and C-banding (Fig. 1d) procedures it can be clearly seen that the two banding patterns are very distinct. The mechanisms responsible for the differences in the two banding patterns are beyond the scope of this paper, but the procedures described here are consistent and highly reproducible.

The HKG-banding technique was first described by Shang et al. (1988) to analyse heterochromatin diversity in wheat chromosomes. Although they obtained excellent results with wheat, we had to adapt the banding technique for maize chromosomes. The major modifications were as follows: (i) fixation of root tips with methanol-acetic acid (3:1); enzymatic maceration to produce a cellular suspension and the dropping airdrying technique (these procedures allow maize chromosomes to spread, in the same plane of focus and with better morphology by comparison with the squash technique); (ii) after hydrolysis in $1 \mathrm{~N} \mathrm{HCl}$ at $60^{\circ} \mathrm{C}$ for 4-6 min, the slides were washed with 0.9 per cent $\mathrm{NaCl}, 70$ per cent ethanol and fast dried on a hot-plate, instead of being dried for 4-6 h; (iii) the concentration of the $\mathrm{KOH}$ solution $(0.06 \mathrm{~N})$ and the duration of the alkaline treatment $(8-12 \mathrm{~h})$ was optimized for maize chromosomes; (iv) after the treatment with $\mathrm{KOH}$, the slides were washed with 70 per cent ethanol, absolute ethanol and methanol-acetic acid $(8: 1)$ instead of
Sorenson's buffer. This last modification is essential in order to obtain a higher contrast and differentiation of the bands by Giemsa staining.

An improved, practical and short C-banding procedure was developed based on the observation that after air-drying it is possible to get good C-banding resolution with a short barium hydroxide treatment and without the 2 XSSC step.

Chow \& Larter (1981) described a C-banding technique modified for maize root tip chromosomes, using Leishman staining, to produce a complete set of centromeric and interstitial bands in addition to those revealed by other authors. However, their figures showed centromeric bands only for chromosomes 1,2 and 5 ; additionally no clearly defined interstitial bands were shown. The presence of centromeric bands in maize chromosomes was also reported by Hadlaczky \& Kalman (1975), Ward (1980), Yun-Chun \& Li-Hua (1983), Khuong \& Schubert (1986) and Aguiar-Perecin (1985), but their figures did not clearly show the presence of these bands. These authors mention technical difficulties or staining inconsistency of centromeric bands with the $\mathrm{C}$-banding technique. However, with the HKG-banding pattern described in this paper, the centromeric bands as well as the intercalary bands (Fig. 1a,b,c) can be clearly seen in all chromosomes of the complement.

From a cytological viewpoint, the new heterochromatin patterns we obtained through the HKG- and $\mathrm{C}$-banding techniques clearly demonstrate that knob heterochromatin and centromeric heterochromatin of A chromosomes in maize are different types of heterochromatin. This has also been suggested by Hadlaczky \& Kalman (1975), Ward (1980) and Aguiar-Perecin (1985), based on the C-banding pattern only.

The intercalary HKG band adjacent to the knob which was demonstrated in the present paper may be another type of heterochromatin closely associated with the knob heterochromatin. The fact that this band responded positively to $\mathrm{HKG}$ treatment exactly like the centromeric band suggests that both types of heterochromatin may have similar repetitive DNA sequences. It has been reported (Viotti et al., 1985) that the neocentric activity of knobs is a consequence of DNA composition of knob heterochromatin which may have one or more centromeric sequences interspersed with the highly repeated $\mathrm{H}_{2}$ sequences. According to our results, the HKG knob-adjacent band is a strong candidate for heterochromatin having similar sequences to those of centromeric type. Although this band has been revealed in an intercalary position and adjacent to the knob, it was not possible to determine whether it is an integral portion of the heterochromatin block of the knob or not. 
The results presented in this study demonstrate that our modified HKG-banding technique is able to reveal centromeric, intercalary and intercalary knob-adjacent bands, probably corresponding to the same type of heterochromatin. The C-banding technique, on the other hand, shows bands corresponding to knobs, probably staining only the $\mathrm{H}_{2}$ portion of the knob heterochromatin.

The banding patterns obtained with the two techniques used in the present study re-open the discussion about heterochromatin classification and its distribution in maize somatic chromosomes. Additionally, our results open new possibilities for research on heterochromatin diversity at the cytological level among different maize inbred lines.

\section{Acknowledgement}

The study was supported by the Fundação de Amparo à Pesquisa do Estado de Minas Gerais (FAPEMIG, BRAZIL).

\section{References}

AGUIAR-PERECIN, M. L. R. 1985. C-banding in maize. I. Band Patterns. Caryologia, 38, 23-30.

CARLSON, w. R. 1988. The cytogenetics of corn. In: Sprague, G. F. and Dudley, J. W. (eds) Corn and Corn Improvement.
ASA Monograph. American Society of Agronomy, WI, pp. 259-344.

CHOW, C. AND laRTer, E. N. 1981. Centromeric banding in maize. Can. J. Genet. Cytol., 23, 255-258.

HADLACZKY, G. Y. AND KALMAN, L. 1975. Discrimination of homologous chromosomes of maize with Giemsa staining. Heredity, 35, 371-374.

KHUONG, N. T. AND SCHUBERT, I. 1986. Differential staining of mitotic chromosomes from inbred lines and hybrids of Zea mays L. Kulturpflanze, 34, 195-205.

MASTENBRoEK, I. AND DE WET, J. M. J. 1983. Chromosome C-banding of Zea mays and its closest relatives. Can. J. Genet. Cytol., 25, 203-209.

PEACOCK, W. J., DENNIS, E. S., RHOADES, M. M. AND PRYOR, A. J. 1981. Highly repeated DNA sequence limited to knob heterochromatin in maize. Proc. Natl. Acad. Sci. U.S.A., 78, 4490-4494.

SHANG, M. X., JACKSON, R. C. AND NGUYEN, H. T. 1988. Heterochromatin diversity and chromosome morphology in wheats analyzed by the HKG banding technique. Genome, 30, 956-965.

VIOTTI, A., PRIVITERA, E., SAla, E. AND POGNA, N. 1985. Distribution and clustering of two highly repeated sequences in the $\mathrm{A}$ and $\mathrm{B}$ chromosomes of maize. Theor. Appl. Genet., 70, 234-239.

YUN-CHUN, S. AND LI-HUA, L. 1983. Comparisons between C-banding patterns on root tip chromosomes of different cultivars in maize (Zea mays). Acta Botanica Sinica, 25, 40-45.

WARD, E. J. 1980. Banding patterns in maize mitotic chromosomes. Can. J. Genet. Cytol., 22, 61-67. 